\title{
CONF- $960894-2$ \\ Accelerated Life-Time Testing and Resistance Degradation of Thin-Film Decoupling Capacitors
}

\author{
Husam Al-Shareef and Duane Dimos \\ Sandia National Laboratories \\ Albuquerque, NM 87185
}

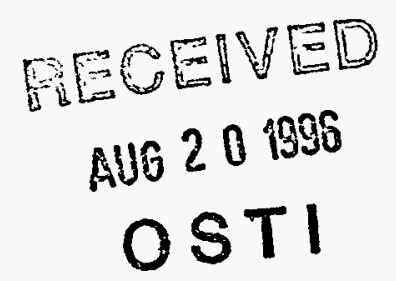

Abstract - Resistance degradation in PZT thin-film capacitors has been studied as a function of applied voltage, temperature, and film composition. It is found that the mean-time-to-failure (life-time or $t_{f}$ ) of the capacitors shows a power law dependence on applied voltage of the form $t_{f} \propto V^{-n}(n \sim 4-5)$. The capacitor life-time also exhibits a temperature dependence of the form $t_{\mathrm{f}} \propto \exp \left(E_{d} / k T\right)$, with an activation energy of $\sim 0.8 \mathrm{eV}$. The steady-state leakage current in these samples appears to be bulk controlled. The voltage, temperature, and polarity dependence of the leakage current collectively suggest a leakage current mechanism most similar to a Frenkel-Poole process. The life-time and leakage current of the $\mathrm{Nb}$-doped PZT films are superior to the undoped PZT films. This result can be explained based on the point-defect chemistry of the PZT system. Finally, the results indicate that the $\mathrm{Nb}$ doped PZT films meet the essential requirements for decoupling capacitor applications.

\section{INTRODUCTION}

Multilayer ceramic capacitors (MLCs) are typically used in signal decoupling applications. However, as the operating frequency of many systems increases, improvement in signal decoupling will be required since the inductance associated with current surface-mount MLCs and their interconnection to the IC will limit the operational frequency. One strategy which has been suggested to improve signal decoupling in highspeed systems, such as multichip modules (MCMs), is to develop low-inductance, thin-film capacitors which can be integrated into the packages $[1,2,3]$.

Thin-film capacitors must meet essentially the same requirements as multilayer ceramic capacitors which they are expected to replace. These requirements include a high dielectric constant, low dissipation factor, low leakage resistance, and acceptable capacitor life-time. Several high permittivity materials have been made in thin-film form; these include perovskite-type oxides such as $\mathrm{Pb}(\mathrm{Zr}, \mathrm{Ti}) \mathrm{O}_{3}(\mathrm{PZT}), \mathrm{Pb}(\mathrm{Mg}, \mathrm{Nb}) \mathrm{O}_{3}(\mathrm{PMN})$, and
(Ba,Sr) $\mathrm{TiO}_{3}$ (BST). Previous studies have shown that the leakage resistance and dielectric properties (e.g., $\varepsilon$ and $\tan \delta$ ) of several PZT-based thin-film compositions are clearly good enough for decoupling capacitor applications [3]. However, little has been done to evaluate the resistance degradation characteristics of PZT thin-film decoupling capacitors.

Resistance degradation, the slow increase of the leakage current under simultaneous temperature and dc voltage stress, has always been a primary failure concern in MLCs. It is therefore essential to study the resistance degradation characteristics of high permittivity thin-films. Such a study will allow a more thorough evaluation of the potential for thinfilm capacitors to replace existing MLCs. Thus, the goal of this work is to determine the resistance degradation and life-time properties of selected PZT compositions under a variety of testing conditions.

\section{EXPERIMENT}

The capacitors were fabricated by first depositing PZT thin-films on Pt-coated $\mathrm{SiO}_{2} / \mathrm{Si}$ substrates using a solution deposition process which has been previously described[4]. Films were synthesized by spin-coating multiple layers of a precursor solution onto the $\mathrm{Pt}$-coated $\mathrm{SiO}_{2} / \mathrm{Si}$ substrate. Each layer was spin-coated at $3000 \mathrm{rpm}$ for $30 \mathrm{sec}$ using a photoresist spinner, and then heat treated on a hot plate at $300^{\circ} \mathrm{C}$ for $5 \mathrm{~min}$. After 4 layers had been deposited, the films were crystallized at $650^{\circ} \mathrm{C}$ for $1 / 2 \mathrm{hr}$ in air. This process was repeated to produce films which are nominally $0.8 \mu \mathrm{m}$ thick. Next, Pt top electrodes, $2.5 \mathrm{~mm}$ in diameter, were deposited on the films using magnetron sputter-deposition. The entire capacitor structure was then annealed at $550^{\circ} \mathrm{C}$ for $1 / 2$ hour in air prior to performing any electrical tests. This annealing step was found to result in more consistent and reproducible leakage currents.

Two compositions were investigated in this study, namely, PNZT 3/40/60 (i.e., Nb-doped PZT) and PZT 40/60. These two compositions are the first 


\section{DISCLAIMER}

Portions of this document may be illegible in electronic image products. Images are produced from the best available original document. 


\section{DISCLAIMER}

This report was prepared as an account of work sponsored by an agency of the United States Government. Neither the United States Government nor any agency thereof, nor any of their employees, makes any warranty, express or implied, or assumes any legal liability or responsibility for the accuracy, completeness, or usefulness of any information, apparatus, product, or process disclosed, or represents that its use would not infringe privately owned rights. Reference herein to any specific commercial product, process, or service by trade name, trademark, manufacturer, or otherwise does not necessarily constitute or imply its endorsement, recommendation, or favoring by the United States Government or any agency thereof. The views and opinions of authors expressed herein do not necessarily state or reflect those of the United States Government or any agency thereof. 
to be investigated among several compositions and electrode systems which we plan to study. The resistance degradation and life-time measurements were performed on these samples by monitoring the current-time response at various dc voltages and temperatures. Unless otherwise stated, the top electrode was biased positively with respect to the bottom electrode. In order to improve the statistical significance of the data, three capacitors were tested simultaneously for every set of testing conditions. This was achieved by using three independent Keithley 236 source-measure units (SMUs). The samples were heated using a Signatone $6 "$ hot stage controlled by a dc power supply to minimize noise. The whole setup was computer controlled using an automated data acquisition system. The samples were tested using temperatures in the range of 100 $170^{\circ} \mathrm{C}$ and voltages in the range $8-45 \mathrm{~V}$.

\section{RESULTS}

\section{A. Resistance Degradation \& Life-Time}

The effect of applied voltage on the current-time response of PNZT 3/40/60 capacitors is shown in Fig. 1. The current decays until a steady-state value is reached followed by a rather abrupt current increase. The abruptness of the current increase depends on the sign of applied voltage. It was generally observed that when the top electrode was biased negatively with respect to the bottom electrode, the current increase was more gradual.

Fig. 2 shows the voltage dependence of the capacitor mean-time-to-failure (life-time or $t_{f}$ ). In this study, capacitor life-time is defined as the time at which the current through the capacitor increases by one order of magnitude from its minimum steadystate value. While other criteria can be used to define the life-time of a capacitor [5-6], this definition, as pointed out by Waser et al.[7], has the advantage of keeping the life-time $\left(t_{f}\right)$ independent of the absolute value of the current density. Fig. 2 indicates that the life-time $\left(t_{f}\right)$ of these capacitors follows an empirical power law dependence on voltage that is given by:

$t_{f} \propto V^{-n}$

where $V$ is the applied voltage and $n$ is a constant with a value of 4-5, depending on film composition. This behavior is qualitatively similar to what has been typically observed in multilayer ceramic capacitors (MLCs) although the value of $n$ is larger for these films $(\mathrm{n} \sim 2-3$ for MLCs)[5].

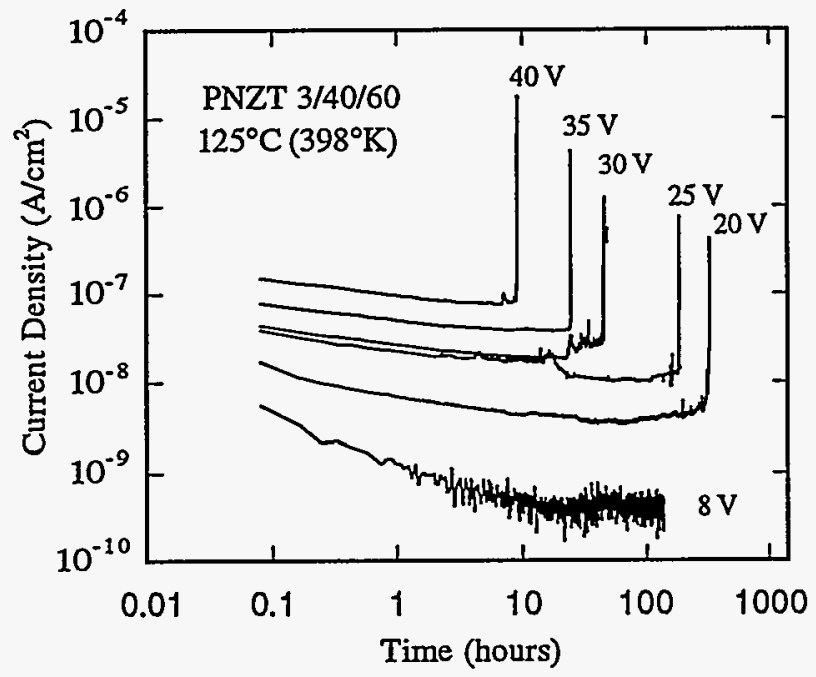

Fig.1. Current-time characteristics of PNZT 3/40/60 thin films as a function of applied voltage.

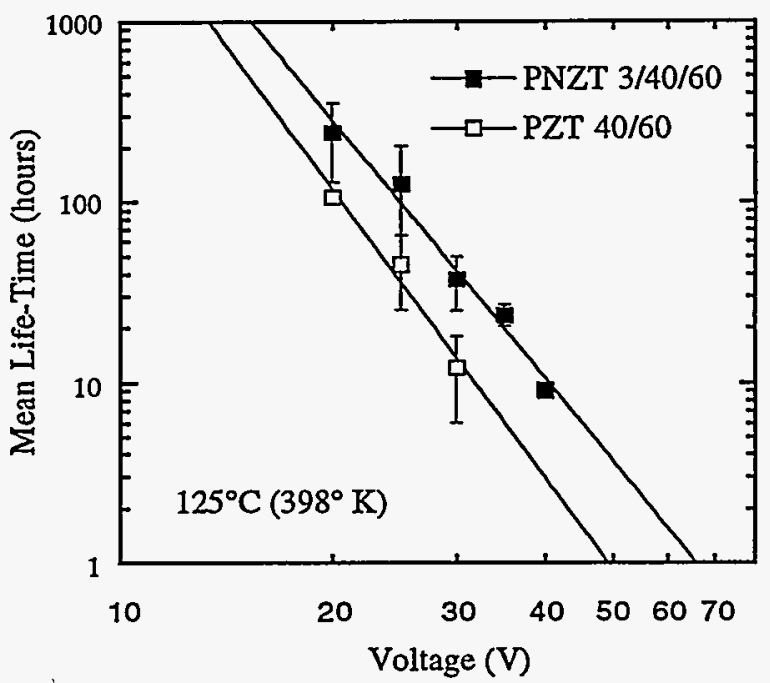

Fig. 2. Dependence of capacitor mean life-time on applied voltage and film composition.

Fig. 2 also shows that the $\mathrm{Nb}$-doped films have longer life-times than undoped PZT $40 / 60$ films. For instance, the life-time of the PNZT 3/40/60 films at $30 \mathrm{~V}$ is over $30 \mathrm{hrs}$, while the life-time of the PZT $40 / 60$ films at the same voltage is about $10 \mathrm{hrs}$.

Fig. 3 shows the temperature dependence of the mean life-time of the same capacitors at two different voltages. It can be seen from Fig. 3 that the life-time $\left(t_{f}\right)$ follows an Arrhenius-type behavior of the form:

$t_{f} \propto \exp \left(E_{d} / k T\right)$

where $E_{a}$ is the activation energy, $k$ is Boltzmann's constant, and $T$ is the temperature in $(\mathrm{K})$. The 


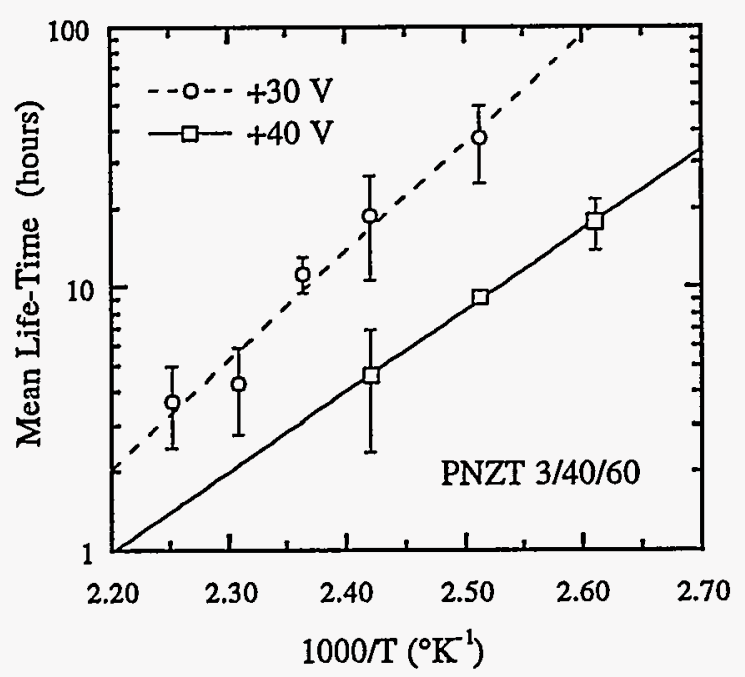

Fig. 3. Temperature dependence of the life-time of PNZT 3/40/60 thin-films at 30 and $40 \mathrm{~V}$.

activation energy calculated from Fig. 3 is voltage dependent and is $\sim 0.82 \mathrm{eV}$ for the measurements performed at $+30 \mathrm{~V}$. This value is somewhat smaller than the activation energies reported for bulk MLCs $(\sim 1 \mathrm{eV})[6,8]$. However, a lower activation energy for degradation has also been observed in other perovskite-type thin films such as $(\mathrm{Ba}, \mathrm{Sr}) \mathrm{TiO}_{3}[8]$.

\section{B. Leakage Current}

The leakage current in these samples was also studied as a function of dc voltage, temperature, composition and polarity. Fig. 4, shows the voltage and composition dependence of the steady state leakage current for PNZT 3/40/60 and PZT 40/60 films. The data is plotted as a function of $\sqrt{ } V$, since this gives the best fit to the leakage data. Fig.4 also shows that the steady-state leakage current of the $\mathrm{Nb}$-doped films is at least three orders of magnitude lower than that of undoped PZT films. In addition, the leakage current shows negligible polarity dependence.

Fig. 5 shows the temperature dependence of the steady-state leakage current at two voltages. The data fit an Arrhenius-type behavior very well. The effective activation energy calculated from Fig. 5 is $0.9-1.0 \mathrm{eV}$.

\section{DISCUSSION}

The data clearly indicate that the life-time and steady-state leakage current of the Nb-doped PZT

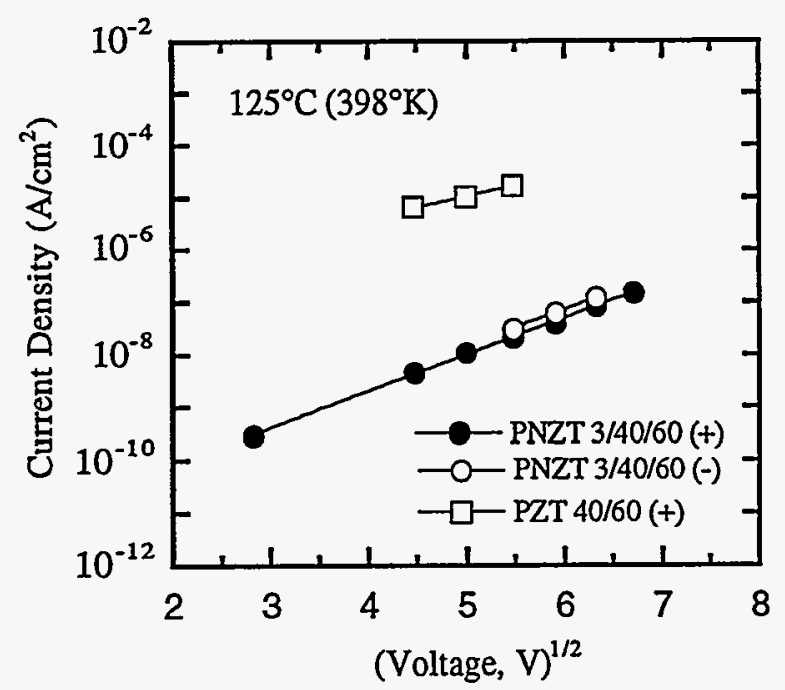

Fig. 4. Steady-state leakage current density vs $V^{1 / 2}$. The $(+)$ and $(-)$ indicate the sign of the top electrode.

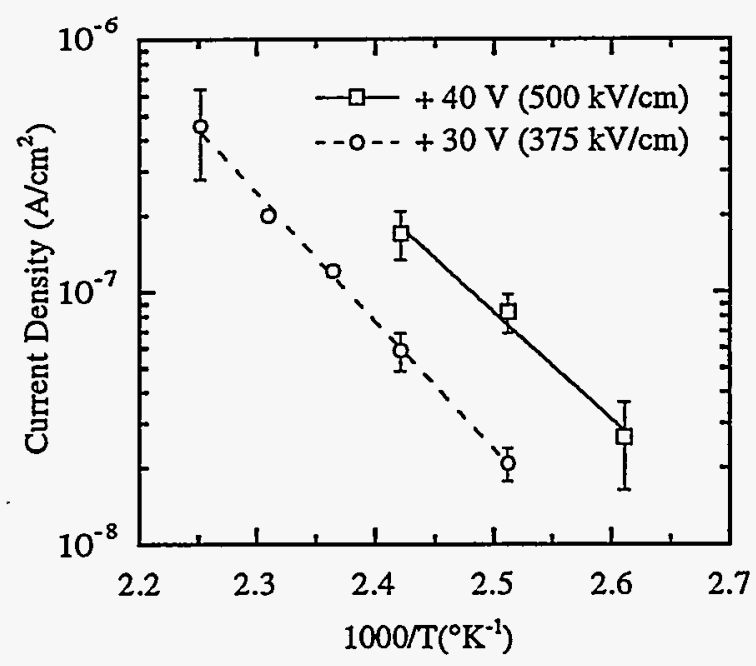

Fig. 5. Temperature dependence of the steady-state leakage current of PNZT 3/40/60 capacitors.

$3 / 40 / 60$ films are superior to the nominally undoped PZT 40/60 films. These results can be explained based on the defect chemistry of the PZT system, a brief review of which is presented below.

It is well-known that nominally undoped PZT compositions exhibit p-type conduction due to the presence of unintentionally-introduced acceptor species[9]. The dominant acceptor species in PZT are expected to be transition-metal impurities and $\mathrm{Pb}$ vacancies. The lead vacancies are associated with $\mathrm{PbO}$ volatilization during elevated temperature processing[9]. Both lead loss and the presence of unintentional acceptor impurities can generate negatively charged defects. These negatively charged acceptors must be balanced by positively 
charged defects to maintain charge neutrality. In nominally undoped PZT, considering only the major defects, the charge neutrality condition is given by:

$2\left[\mathrm{~V}_{\mathrm{O}}{ }^{\circ}\right]+\left[\mathrm{h}^{\circ}\right]=2\left[\mathrm{~V}_{\mathrm{Pb}}{ }^{\prime \prime}\right]+\left[\mathrm{A}_{\mathrm{B}}\right]$

where [] denotes concentration, $\mathrm{h}^{-}$is an electron hole, $A_{B}{ }^{\prime}$ is an acceptor impurity such as Fe on a $B$ site, and $\mathrm{V}_{\mathrm{O}}{ }^{*}$ and $\mathrm{V}_{\mathrm{Pb}}$ " are oxygen and lead vacancies, respectively. With donor doping, e.g. $\mathrm{Nb}$, Eq. (3) is modified and is given by:

$\left[\mathrm{Nb}_{\mathrm{B}}{ }^{\circ}\right]+2\left[\mathrm{~V}_{\mathrm{O}}{ }^{*}\right]+\left[\mathrm{h}^{\circ}\right]=2\left[\mathrm{~V}_{\mathrm{Pb}}{ }^{\circ}\right]+\left[\mathrm{A}_{\mathrm{B}}{ }^{\prime}\right]$

where $\mathrm{Nb}_{\mathrm{B}}{ }^{\circ}$ is a $\mathrm{Nb}^{+5}$ ion on a B-site ( $\mathrm{Zr}$ or Ti site). The $\mathrm{Nb}_{\mathrm{B}}$ defects can compensate the charge due to $\mathrm{V}_{\mathrm{Pb}}$ " and $\mathrm{A}_{\mathrm{B}}$ ', leading to a dramatic decrease in the concentration of $h$ and $V_{0}{ }^{\circ}$.

The superior resistance degradation and leakage current of the $\mathrm{Nb}$-doped PZT films can be explained based on the defect-chemistry concepts outlined above. Most resistance degradation models in perovskite-type oxides are based on the premise that oxygen vacancies $\left(V_{0}^{*}\right)$ play an essential role in the degradation process[7]. Due to space limitation, these models will not be reviewed here, but the reader is referred to reference [7] for a good review. Based on these models, one expects resistance degradation to be more pronounced in systems which have a relatively large concentration of oxygen vacancies. Since, Nb-doped PZT films are expected to have lower [ $\left.\mathrm{V}_{\mathrm{o}}{ }^{\circ}\right]$, they exhibit superior resistance degradation and longer life-times compared to nominally undoped PZT films.

One specification that is typically used to evaluate MLCs is that they must have a life-time in excess of 1000 hours when tested at $125^{\circ} \mathrm{C}$ and twice the operating voltage. The operating voltage in digital decoupling applications is typically around $5 \mathrm{~V}$, although this value will continue to decrease with new generations of devices. By extrapolating the data in Fig. 2 to 1000 hours, it can be seen that the PNZT 3/40/60 and PZT 40/60 films should allow 6 and $8 \mathrm{~V}$ operation, respectively. However, as Fig. 4 shows, the leakage current of the PZT $40 / 60$ films is probably too high for decoupling applications.

The difference in the leakage current between $\mathrm{Nb}$ doped and undoped PZT films can be accounted for by considering defects which are expected to increase conductivity of the PZT system. These include $\mathrm{h}^{\circ}$ and $\mathrm{V}_{\mathrm{o}}{ }^{\circ}$ since they are expected to be mobile over the investigated temperature range[7].
However, the steady-state leakage current in these films should be mainly electronic, since metal electrodes will block any mobile ionic defects such as $\mathrm{V}_{\mathrm{o}}{ }^{\circ}$. Thus, it is very likely that the lowering of leakage current due to $\mathrm{Nb}$-doping occurs as a result decreasing the hole concentration. A similar result has been reported for La-doped PZT films[10].

These defect chemistry arguments assume that the steady-state leakage current in these films is bulkcontrolled. However, the leakage current can also be controlled by other processes such as carrier emission/injection from either trapping centers in the film or over the film/electrode interface. The leakage current mechanisms commonly reported in dielectric thin films include Ohmic conduction (usually at low fields), space-charge limited current (SCLC), Schottky emission, and the Frenkel-Poole effect [11]. One must be careful since more than one mechanism may be operating at one time, which often makes it difficult to fit data to a single process.

The I-V characteristics of our films indicate that steady-state conduction in this temperature and voltage range is non-Ohmic. The best fit for the current-voltage relation of these films was obtained for $\ln (J) \nu s V^{1 / 2}$ as shown in Fig. 4. This usually indicates that the leakage current is controlled by either a Schottky-like process (i.e, emission from the electrode into the conduction band of the dielectric over a barrier) or a Frenkel-Poole process (i.e, field-dependent thermionic emission of carriers from trapping centers in the bulk of the film)[11]. by:

The Schottky-controlled leakage current is given

$J=A * T^{2} \exp \left(\frac{a \sqrt{E}-\Phi_{B}}{k T}\right)$

where $A^{*}$ is the effective Richardson's constant, $T$ is temperature, $a$ is a constant, $E$ is the applied electric field, and $\Phi_{B}$ is the barrier height[11]. In comparison, the leakage current according to the Frenkel-Poole process is given by:

$J=A_{0} E \exp \left(\frac{\beta \sqrt{E}-Q}{k T}\right)$

where $A_{0}$ and $\beta$ are constants, and $Q$ is the depth of the trap potential well[11].

It is unlikely that the steady-state current in these films is controlled by a Schottky-like process for two reasons. First, the top and bottom electrode/film 
interfaces underwent very different thermal cycling conditions. This implies that the interface states and therefore the effective barrier height should be different for the top and bottom interfaces. However, the leakage current is independent of voltage polarity, which suggests that leakage current is more likely bulk than interface controlled. Secondly, according to Eq. (5) a plot of $\ln \left(J / T^{2}\right) v s$ $I / T$ should be linear for a Schottky-like process. However, we find that the $1 / T^{2}$ dependence (not shown) is weak in these films.

The Frenkel-Poole mechanism is a bulk process and would explain both the linearity of the $\ln (J) v s$ $V^{1 / 2}$ and the lack of polarity dependence shown in Fig. 4[11]. Fig. 5 shows a plot of leakage current vs reciprocal temperature for the $\mathrm{Nb}$-doped films (a plot of $\ln (J / E)$ vs $1 / T$ gives an equally good linear fit, as predicted by Eq.6). Fitting the data in Fig. 5 to an equation of the form $J \sim \exp \left(-E_{d} / k T\right)$ gives an activation energy of $1.0 \mathrm{eV}$ for $30 \mathrm{~V}$. In comparison, fitting the same data to the Frenkel-Poole model, Eq.(6), gives an activation energy (Q) of $1.3 \mathrm{eV}$. The reason for this difference is that the FrenkelPoole model assumes a field-dependent thermallyactivated process, while the expression J $J \exp (-$ $\left.E_{d} / T\right)$ does not. In fact, the trap depth (Q) in Eq. (6) is simply the sum of $\beta \sqrt{ } E$ and $E_{a}$.

These results coupled with the defect-chemistry discussion presented earlier suggest that the steadystate leakage current in these films is most likely controlled by thermally-activated, field-dependent emission of trapped holes $\left(h^{\circ}\right)$. Possible trapping centers for the holes include lead vacancies $\left(V_{\mathrm{Pb}}{ }^{\prime}\right)$ and transition metal impurities $\left(\mathrm{A}_{\mathrm{B}}{ }^{\prime}\right)$, which are known to form deep traps in PZT[12].

\section{CONCLUSIONS}

This main findings of this work can be summarized as follows:

- The PZT thin films meet an essential life-time requirement for decoupling capacitor applications. Specifically, the films exhibit a life-time of $\sim 1000$ hours at $125^{\circ} \mathrm{C}$ and an operating voltage of $8 \mathrm{~V}$ for the $\mathrm{Nb}$-doped films and $6 \mathrm{~V}$ for the undoped films.

- The leakage current of the undoped PZT films is at least three orders of magnitude higher than that of $\mathrm{Nb}$-doped films. Thus, undoped PZT films may have too high a leakage current to be usable in decoupling applications. Donor doping of PZT may therefore be essential.
- The life-time of the PZT films investigated shows a power-law voltage dependence and an Arrheniustype temperature dependence, which is similar to multilayer ceramic capacitors.

- The leakage current in these films is bulkcontrolled and shows a voltage and temperature dependence typical of a Frenkel-Poole process.

\section{ACKNOWLEDGMENTS}

The authors would like to thank Amelia Sanchez and Walter Olson for their excellent technical assistance. This work was performed at Sandia National Laboratories, supported by the U.S. DOE under contract No. DE-AC04-94AL85000.

\section{REFERENCES}

[1] B. K. Gilbert and W. L. Walters, Intl. J. Microcircuits and Elec. Packaging, Vol 15, 171(1992).

[2] R. C. Frye, Proc. 1992 IEEE Multi-Chip Module Conf., 155(1992).

[3] D. Dimos, S. J. Lockwood, R. W. Schwartz, and M. S. Rodgers, IEEE Trans. CPMT-Part A, Vol 18, 174(1995).

[4] R. Schwartz, R. Assink, and T. Headley, Mater. Res. Soc. Symp. Proc., Vol 243, 245(1993).

[5] W.J. Minford, IEEE Trans. Components, Hybrids, and Manuf. Technol., Vol CHMT-5, No.3, 297(1982).

[6] R. Munikoti and P. Dhar, IEEE Trans. Components, Hybrids, and Manuf. Technol., Vol CHMT-11, 342 (1988).

[7] R. Waser, T. Baiatu, and K. H. Hardtl, J. Am. Ceram. Soc., Vol 73, 1645(1990).

[8] K. Numata, Y. Fukuda, K. Aoki, and A. Nishimura, Jpn. J. Appl. Phys., Vol 34, 5245(1995).

[9] D. M. Smyth, Prog. Solid St. Chem, Vol 15, 145(1984). [10] D. Dimos, R. W. Schwartz, and S. J. Lockwood, J. Am. Ceram. Soc., Vol 77,3000(1994).

[11] J.J. O'Dwyer, The Theory of Electrical Condution and Breakdown in Solid Dielectrics, Clarendon Press, Oxford (1973). [12] M. V. Raymond, and D. M. Smyth, Integr. Ferroelectr., Vol 4, 145(1994). 